\title{
Bosonic D-branes at finite temperature with an external field
}

\author{
M. C. B. Abdalla, A. L. Gadelha, and I. V. Vancea* \\ Instituto de Física Teórica, Universidade Estadual Paulista, Rua Pamplona 145, 01405-900, São Paulo, SP, Brazil
}

(Received 10 May 2001; published 26 September 2001)

\begin{abstract}
Bosonic boundary states at finite temperature are constructed as solutions of boundary conditions at $T \neq 0$ for bosonic open strings with a constant gauge field $F_{a b}$ coupled to the boundary. The construction is done in the framework of thermo field dynamics where a thermal Bogoliubov transformation maps states and operators to finite temperature. Boundary states are given in terms of states from the direct product space between the Fock space of the closed string and another identical copy of it. By analogy with zero temperature, the boundary states have the interpretation of $\mathrm{D} p$-branes at finite temperature. The boundary conditions admit two different solutions. The entropy of the closed string in a $\mathrm{D} p$-brane state is computed and analyzed. It is interpreted as the entropy of the $\mathrm{D} p$-brane at finite temperature.
\end{abstract}

DOI: 10.1103/PhysRevD.64.086005

PACS number(s): 11.25. $-\mathrm{w}, 11.27 .+\mathrm{d}$

\section{INTRODUCTION}

There have been various reasons why string theories at finite temperature have been considered an interesting subject [1]. In the past, it was suggested in [2] that high-energy, fixed-angle scattering could give some information about the physics of string theory such as, for example, the spontaneously broken-symmetry group. The properties of an ideal gas of superstrings were used to model the cosmology of the early universe in Refs. [4-9]. The high-energy behavior of the thermal ensemble of the supersymmetric string theory showed that the underlying degrees of freedom of string theory are less than known in relativistic field theories and that there is a first-order transition at the Hagedorn temperature in an ideal gas of strings [3]. To investigate the behavior of string theory near the Hagedorn temperature, a real-time finite-temperature interacting strings technique was developed in Refs. [10-12].

Along another line of development, it was proved that the $\mathrm{D} p$-branes belong to the string spectra and couple with Ramond-Ramond fields [13]. The D $p$-branes play a crucial role in understanding string theory and its connection with field theories and gravity $[14,15]$. Also, they have been used to understand the statistical properties of various systems. The energy-entropy relation, the Hawking temperature, and the Hagedorn transition of extreme, near-extreme, and Schwarzschild black holes were calculated from the statistics of the Boltzman gas of free D0-branes and from the counting of microstates in certain conformal field theories [16-32]. However, despite the relative knowledge of strings at finite temperature and the positive results obtained from $\mathrm{D} p$-brane ensembles, not much is known about the statistical properties of the D $p$-branes (but see Refs. [33-41]).

In the absence of background fields, a $\mathrm{D} p$-brane has a microscopic description as a boundary state in the Fock space of the perturbative closed string where the brane is represented as a particular superposition of some coherent states on the boundary of the cylinder. This description con-

\footnotetext{
*On leave from Babes-Bolyai University of Cluj.
}

tinues to hold when a a gauge field is turned on [42-50]. Since the $\mathrm{D} p$-brane is written in terms of string operators acting on the vacuum, one can apply the thermo field method [51] to construct the boundary states at finite temperature. Since the states should belong to the physical spectrum of the string, the contribution of ghosts should be taken into account [55]. The thermo field dynamics was used previously to study the renormalization of open bosonic strings at finite temperature. The renormalizability was proved and the model was showed to be compatible with the thermal Veneziano amplitude in Refs. [52,53]. The global phase structure of bosonic-thermal-string ensemble and its connection with the thermal string amplitude was described in Ref. [54].

In a previous paper [56], the Bogoliubov transformations of the thermo field were used to construct the bosonic string at finite temperature in a flat Minkowski spacetime and in the light-cone gauge. The boundary conditions necessary to define the $\mathrm{D} p$-branes at finite temperature as states in the Fock space were obtained, and it was shown that the corresponding equations admit solutions that can be interpreted as bosonic branes. Some particular solutions that reduce to the known $\mathrm{D} p$-branes at $T=0$ were also given. However, the analysis was not complete.

The aim of this paper is to report on some new solutions of the bosonic boundary conditions at $T \neq 0$ and generalize the construction to a nontrivial background in which an $S O(1, p)$ photon $A^{a}$ is present on the world volume of the brane. To keep the program simple, we consider that the photon does not depend on the temperature, although the most general case can be written down by recalling that the photon belongs to the spectrum of the open strings that ends on the brane. We also give the solutions of the boundary conditions that are a superposition of closed string states at finite temperature. By analogy with $T=0$, we interpret the boundary states as $\mathrm{D} p$-branes at $T \neq 0$. Within the framework of the thermo field, it is possible to compute the entropy as the expectation value of the entropy operator. We compute the value of this entropy in a $\mathrm{D} p$-brane state and we show that it has the following behavior. In the limit of low temperatures $T \rightarrow 0$, the contribution of the closed string oscillators diverges and one is left with a series in the powers of the 
$(I-\mathbb{F} / \mathbb{I}+\mathbb{F})$ where $F$ is the gauge field. However, in the limit when $T \rightarrow \infty$, the oscillator contribution is proportional to $\log (-1)$ which does not make sense. This might be a consequence of the fact that above a critical temperature (the Hagedorn temperature) the notion of temperature breaks down in string theory. If this is the case, even if formally we can construct the boundary states at any temperature, they do not make sense at temperatures where the string model ceases to make sense. We do not have a good explanation of the general behavior of the entropy. It is premature to say, even if the construction of these states is acceptable, if the behavior of the entropy signals that the bosonic D $p$-branes cannot be described as acceptable states in the Fock space of closed bosonic string theory. However, before drawing such of conclusions, deeper study of the model should be performed and we refer the reader to Ref. [57] for further discussions of these problems.

The paper is organized as follows. In Sec. II we construct the closed string at finite temperature in the thermo field approach and give the boundary conditions and the $\mathrm{D} p$-brane at $T \neq 0$. In Sec. III we compute the entropy of $\mathrm{D} p$-brane states. Section IV is devoted to discussions. The basic relations of thermo field dynamics, which have been used throughout the text, are presented in the Appendix.

\section{BOUNDARY STATES AT FINITE TEMPERATURE WITH AN EXTERNAL FIELD}

In this section we construct the boundary states of the bosonic closed strings in the presence of an open string $S O(1, p)$ vector field at finite temperature. We then give the solutions of the corresponding equations.

\section{A. Closed strings at $T \neq 0$}

Consider the bosonic open string on flat Minkowski spacetime in the presence of a bosonic and rigid $\mathrm{D} p$-brane located at the boundary $\sigma=0$ of the world sheet. The $U(1)$ charges at the endpoints of the string generate a vector field $A^{a}$, that lives on the world volume of the brane. To avoid dealing with ghosts, we choose to work in the light-cone gauge $X^{0} \pm X^{25}$. Then $a, b, \ldots=1, \ldots, p$ denote the indices of the tangential coordinates to the world volume. For the transversal coordinate we use the indices $i, j, \ldots=p$ $+1, \ldots, 24$. The spacetime indices are labeled by $\mu, \nu, \ldots$ $=1, \ldots, 24$. By interpreting the one-loop diagram in open string amplitude as the tree-level diagram in the closed string channel, one can give a microscopic description of the $\mathrm{D} p$-brane as a state in the Fock space of the free closed string. This state is defined by $p+1$ Neumann boundary conditions and 25- $p$ Dirichlet boundary conditions acting on a vector of the Fock space. At $T=0$ and in the presence of the $S O(1, p)$ photon, the corresponding relations, written in terms of string modes, are given by

$$
\begin{array}{r}
{\left[(\mathbb{I}+\hat{\mathbb{F}})_{b}^{a} \alpha_{n}^{b}+(\mathbb{I}-\hat{\mathbb{F}})_{b}^{a} \beta_{-n}^{b}\right]|B\rangle=0,} \\
\left(\alpha_{n}^{i}-\beta_{-n}^{i}\right)|B\rangle=0,
\end{array}
$$

$$
\hat{p}^{a}|B\rangle=\left(\hat{q}^{i}-y^{i}\right)|B\rangle=0,
$$

for any $n>0$, where $\alpha_{n}^{a}$ and $\beta_{n}^{b}$ are, respectively, the rightand left-moving modes of the closed string. $q^{i}$ and $\hat{p}^{a}$ are components of the coordinate of the center-of-mass and its momenta, respectively, and $y^{i}$ are the coordinates of the $\mathrm{D} p$-brane in the transverse space. For the scaled gauge field we use the notation

$$
\widehat{\mathbb{F}}_{a b}=2 \pi \alpha^{\prime} F_{a b},
$$

where $F_{a b}$ is constant. The state that satisfies the boundary conditions (1) is denoted by $|B\rangle$. This state belongs to the bosonic Fock space. In a covariant gauge, one should take into account the ghost contribution, and then the brane state factorizes in a term that satisfies Eq. (1) and another one that contains the ghost contribution. The solution to Eq. (1) is a superposition of coherent states of the following form $[49,50]$ :

$$
\begin{aligned}
|B\rangle & =\exp \left[-\sum_{n=1}^{\infty} \alpha_{-n}^{\mu} M_{\mu \nu} \beta_{-n}^{\nu}\right]|B\rangle^{(0)}, \\
|B\rangle^{(0)} & =N_{p} \delta^{\left(d_{\perp}\right)}(\hat{q}-y)|0\rangle,
\end{aligned}
$$

where $N_{p}$ is a normalization constant equal to half of the brane tension and the delta function localizes the state in the trasverse space in the position $q^{i}$. The vacuum state includes a term that is an eigenvector of the momentum operators, which is not written explicitly. The matrix that connects the right and left modes is given by the following relation:

$$
M_{\nu}^{\mu}=\left[\left(\frac{\mathbb{I}-\mathbb{F}}{\mathbb{I}+\mathbb{F}}\right)_{b}^{a} ;-\delta_{j}^{i}\right] .
$$

Our first purpose is to construct the finite-temperature counterpart of the boundary states (1). Since the relations are expressed in terms of bosonic string operators and states, one has to map these objects at $T \neq 0$. A convenient way to do that is by employing thermo field techniques [51], which are suitable for systems that are represented in terms of oscillators. (Some basic relations of this construction are presented in Appendix A.) The thermo field approach was used previously to study strings in Refs. [52-55] and Dp-branes in Ref. [56]. According to the thermo field dynamics, the thermodynamics of the system is described in an enlarged Fock space (which is also true in the path-integral approach) composed by the initial Fock space and an identical copy of it. This is what is meant by "doubling the system." The total thermic system is composed by the original string and its copy, denoted by $\sim$. The two copies are independent and the Fock space is the direct product of them.

In order to implement this construction for the case of the bosonic string [56], we use the oscillator operators for left and right modes

$$
A_{n}^{\mu}=\frac{1}{\sqrt{n}} \alpha_{n}^{\mu}, \quad A_{n}^{\mu \dagger}=\frac{1}{\sqrt{n}} \alpha_{-n}^{\mu},
$$




$$
B_{n}^{\mu}=\frac{1}{\sqrt{n}} \beta_{n}^{\mu}, \quad B_{n}^{\mu \dagger}=\frac{1}{\sqrt{n}} \beta_{-n}^{\mu},
$$

where $n>0$. Identical operators exist for the copy of the system. The two algebras are independent

$$
\begin{aligned}
& {\left[A_{n}^{\mu}, A_{m}^{\nu^{\dagger}}\right]=\left[\widetilde{A}_{n}^{\mu}, \widetilde{A}_{m}^{\nu^{\dagger}}\right]=\delta_{n, m} \eta^{\mu \nu},} \\
& {\left[A_{n}^{\mu}, \widetilde{A}_{m}^{\nu}\right]=\left[A_{n}^{\mu}, \widetilde{A}_{m}^{\nu^{\dagger}}\right]=\left[A_{n}^{\mu}, \widetilde{B}_{m}^{\nu}\right]=\cdots=0 .}
\end{aligned}
$$

The extended Fock space of the total system is given by the direct product of the two Fock spaces of closed strings

$$
\hat{H}=H \otimes \widetilde{H} .
$$

A state from $\hat{H}$ is denoted by $|\quad\rangle\rangle$. The vacuum states of the left and right sectors are direct products of the vacuum states of the string and tilde string

$$
\begin{gathered}
|0\rangle\rangle_{\alpha}=|0\rangle_{\alpha} \otimes \widetilde{|0\rangle_{\alpha}}=|0,0\rangle_{\alpha}, \\
|0\rangle\rangle_{\beta}=|0\rangle_{\beta} \otimes \widetilde{|0\rangle_{\beta}}=|0,0\rangle_{\beta},
\end{gathered}
$$

and the following expressions are equal among them

$$
\begin{aligned}
|0\rangle\rangle & \left.=|0\rangle\rangle_{\alpha}|0\rangle\right\rangle_{\beta}=\left(|0\rangle_{\alpha}|\widetilde{0}\rangle_{\alpha}\right)\left(|0\rangle_{\beta}|\widetilde{0}\rangle_{\beta}\right) \\
& =\left(|0\rangle_{\alpha}|0\rangle_{\beta}\right)\left(|\widetilde{0}\rangle_{\alpha}|\widetilde{0}\rangle_{\beta}\right),
\end{aligned}
$$

where the last equality is a consequence of the fact that the original string and the tilde string are independent. The first line in Eq. (13) shows explicitly the doubling of each oscillator, while the second one shows the string-tilde-string structure of the vacuum state. In order to obtain the fundamental state of the enlarged system, we have to multiply Eq. (13) by $|p\rangle|\tilde{p}\rangle$.

The extended system represents the general framework for studing the thermal properties of the initial one. The finite temperature is introduced by a set of Bogoliubov unitary operators acting on the states, as well as on the operators of the extended system. These operators are constructed for each of the oscillating modes of the two copies, and according to the thermo field construction, their form in the right and left sectors is given by the following relations:

$$
\begin{aligned}
& G_{n}^{\alpha}=-i \theta\left(\beta_{T}\right)\left(A_{n} \widetilde{A}_{n}-A_{n}^{\dagger} \widetilde{A}_{n}^{\dagger}\right), \\
& G_{n}^{\beta}=-i \theta\left(\beta_{T}\right)\left(B_{n} \widetilde{B}_{n}-B_{n}^{\dagger} \widetilde{B}_{n}^{\dagger}\right) .
\end{aligned}
$$

Here, $\beta_{T}=\left(k_{B} T\right)^{-1}$, where $k_{B}$ is the Boltzmann's constant and $\theta$ is a parameter depending on the temperature hyperbolically as

$$
\cosh \theta_{n}\left(\beta_{T}\right)=u_{n}\left(\beta_{T}\right)
$$

where $u_{n}\left(\beta_{T}\right)$ is related to the statistics of the modes. For bosonic oscillators, two hyperbolic trigonometric functions are defined (see the Appendix):

$$
\begin{aligned}
& u_{n}\left(\beta_{T}\right)=\left(1-e^{-\beta_{T} \omega_{n}}\right)^{-1 / 2}, \\
& v_{n}\left(\beta_{T}\right)=\left(e^{\beta_{T} \omega_{n}}-1\right)^{-1 / 2} .
\end{aligned}
$$

Let us look at the properties of the Bogoliubov operators. From the definitions (14) and (15) it is easy to see that they are Hermitian

$$
\left(G_{n}^{\alpha}\right)^{\dagger}=G_{n}^{\alpha}, \quad\left(G_{n}^{\beta}\right)^{\dagger}=G_{n}^{\beta},
$$

and that for negative $n$ the following relation holds:

$$
G_{|n|}^{\alpha}=-G_{-n}^{\alpha}
$$

Since the right- and left-moving modes are independent, the corresponding $G$ operators commute among themselves

$$
\left[G_{n}^{\alpha}, G_{m}^{\alpha}\right]=\left[G_{n}^{\beta}, G_{m}^{\beta}\right]=\left[G_{n}^{\alpha}, G_{m}^{\beta}\right]=0 .
$$

A simple algebra gives the rest of the commutation relations among the $G$ operators and the oscillators

$$
\begin{gathered}
{\left[G_{n}^{\alpha}, A_{n}^{\mu}\right]=-i \theta_{n}\left(\beta_{T}\right) \widetilde{A}_{n}^{\mu \dagger}, \quad\left[G_{n}^{\alpha}, B_{n}^{\mu}\right]=-i \theta_{n}\left(\beta_{T}\right) \widetilde{B}_{n}^{\mu \dagger},} \\
{\left[G_{n}^{\alpha}, A_{n}^{\mu \dagger}\right]=-i \theta_{n}\left(\beta_{T}\right) \widetilde{A}_{n}^{\mu}, \quad\left[G_{n}^{\alpha}, B_{n}^{\mu \dagger}\right]=-i \theta_{n}\left(\beta_{T}\right) \widetilde{B}_{n}^{\mu},} \\
{\left[G_{n}^{\alpha}, \widetilde{A}_{n}^{\mu}\right]=-i \theta_{n}\left(\beta_{T}\right) A_{n}^{\mu \dagger}, \quad\left[G_{n}^{\alpha}, \widetilde{B}_{n}^{\mu}\right]=-i \theta_{n}\left(\beta_{T}\right) B_{n}^{\mu \dagger},} \\
{\left[G_{n}^{\alpha}, \widetilde{A}_{n}^{\mu \dagger}\right]=-i \theta_{n}\left(\beta_{T}\right) A_{n}^{\mu}, \quad\left[G_{n}^{\alpha}, \widetilde{B}_{n}^{\mu \dagger}\right]=-i \theta_{n}\left(\beta_{T}\right) B_{n}^{\mu} .}
\end{gathered}
$$

Let us proceed to the construction of the vacuum state and creation and annihilation operators at $T \neq 0$ following Ref. [51]. By acting on the right and left vacuua at $T=0$ with any of the Bogoliubov operators (14) and (15), the new states, which depend explicitly on the temperature, are obtained:

$$
\begin{aligned}
& \left.\left.\left|0\left(\beta_{T}\right)\right\rangle\right\rangle_{\alpha}=\prod_{n>0} e^{\left.-i G_{n}^{\alpha}|0\rangle\right\rangle_{\alpha}}=\prod_{n>0}\left|0\left(\beta_{T}\right)_{n}\right\rangle\right\rangle_{\alpha}, \\
& \left.\left.\left.\left|0\left(\beta_{T}\right)\right\rangle\right\rangle_{\beta}=\prod_{m>0} e^{-i G_{m}^{\beta}}|0\rangle\right\rangle_{\beta}=\prod_{n>0}\left|0\left(\beta_{T}\right)_{n}\right\rangle\right\rangle_{\beta} .
\end{aligned}
$$

Since the $G$ operators do not mix the left- and right-moving states, one can construct a direct product of the states above

$$
\left.\left.\left.\left|0\left(\beta_{T}\right)\right\rangle\right\rangle=\left|0\left(\beta_{T}\right)\right\rangle\right\rangle_{\alpha}\left|0\left(\beta_{T}\right)\right\rangle\right\rangle_{\beta} .
$$

The Bogoliubov transformations acting on the oscillator operators $\left\{A^{\dagger}, A, \widetilde{A}^{\dagger}, \widetilde{A}\right\}$ and $\left\{B^{\dagger}, B, \widetilde{B}^{\dagger}, \widetilde{B}\right\}$ map them to some new operators that depend on temperature

$$
\begin{aligned}
& A_{n}^{\mu}\left(\beta_{T}\right)=e^{-i G_{n}^{\alpha} A_{n}^{\mu} e^{i G_{n}^{\alpha}},} \quad B_{n}^{\mu}\left(\beta_{T}\right)=e^{-i G_{n}^{\alpha}} B_{n}^{\mu} e^{i G_{n}^{\alpha}}, \\
& \widetilde{A}_{n}^{\mu}\left(\beta_{T}\right)=e^{-i G_{n}^{\alpha}} \widetilde{A}_{n}^{\mu} e^{i G_{n}^{\alpha}}, \quad \widetilde{B}_{n}^{\mu}\left(\beta_{T}\right)=e^{-i G_{n}^{\alpha}} \widetilde{B}_{n}^{\mu} e^{i G_{n}^{\alpha}} .
\end{aligned}
$$


From the properties (19)-(22) one can see that the oscillator operators at finite temperature, can be cast in the following form

$$
\begin{aligned}
& A_{n}^{\mu}\left(\beta_{T}\right)=u_{n}\left(\beta_{T}\right) A_{n}^{\mu}-v_{n}(\beta) \widetilde{A}_{n}^{\mu \dagger}, \\
& B_{n}^{\mu}\left(\beta_{T}\right)=u_{n}\left(\beta_{T}\right) B_{n}^{\mu}-v_{n}(\beta) \widetilde{B}_{n}^{\mu \dagger}, \\
& \widetilde{A}_{n}^{\mu}\left(\beta_{T}\right)=u_{n}\left(\beta_{T}\right) \widetilde{A}_{n}^{\mu}-v_{n}\left(\beta_{T}\right) A_{n}^{\mu \dagger}, \\
& \widetilde{B}_{n}^{\mu}\left(\beta_{T}\right)=u_{n}\left(\beta_{T}\right) \widetilde{B}_{n}^{\mu}-v_{n}\left(\beta_{T}\right) B_{n}^{\mu \dagger} .
\end{aligned}
$$

The notation for the state (25) is now justified. Indeed, this state is annihilated by the finite-temperature operators in both the left and right sectors, and for the string and its copy

$$
\begin{aligned}
& \left.\left.A_{n}^{\mu}\left(\beta_{T}\right)\left|0\left(\beta_{T}\right)\right\rangle\right\rangle_{\alpha}=\widetilde{A}_{n}^{\mu}(\beta)\left|0\left(\beta_{T}\right)\right\rangle\right\rangle_{\alpha}=0, \\
& \left.\left.B_{n}^{\mu}\left(\beta_{T}\right)\left|0\left(\beta_{T}\right)\right\rangle\right\rangle_{\beta}=\widetilde{B}_{n}^{\mu}(\beta)\left|0\left(\beta_{T}\right)\right\rangle\right\rangle_{\beta}=0,
\end{aligned}
$$

which shows that Eq. (25) has the properties of the vacuum state.

The finite-temperature operators statisfy the oscillator algebra for each mode, in each sector and for both copies of the original string and all these algebras are independent. Therefore, the states of the system at finite temperature are obtained by acting on the thermal vacuum (25) with the creation and annihilation operators. These states belong to the total Fock space. The Bogoliubov transformations have picked up another vacuum and other oscillators. However, we should note that the thermal vacuum contains infinitely many quanta of zero temperature operators of all types due to the form of the Bogoliubov operators. By creating a quanta of, say, $A$ type, one of $\widetilde{A}$ type is destroyed which suggests that the second copy of the system be interpreted as a thermal reservatoire [51].

An important question is whether the thermo field construction presented above gives a string theory at $T \neq 0$ or maps it into something else. If we plug the finite temperature operators into a solution of the equations of motion of closed bosonic string, one obtains another solution which depends on $T$ but which mixes the original string and the tilde-string in an obvious way. ${ }^{1}$ One can show that all the properties of the bosonic string at zero temperature are satisfied. In particular, one can construct, from the solutions of the equations of motion, the energy-momentum tensor that has the same form as the one at $T=0$. Then the following operators

$$
L_{m}^{\alpha}\left(\beta_{T}\right)=\frac{1}{2} \sum_{k \in Z} \alpha_{-k}\left(\beta_{T}\right) \alpha_{k+m}\left(\beta_{T}\right),
$$

satisfy the Virasoro algebra, as can be shown by using the properties of the Bogoliubov operators (19)-(22). Therefore, the conformal symmetry is not broken by the present con-

\footnotetext{
${ }^{1}$ This results from the definition of $G$ operators, which do not affect the world-sheet waves but only the Fourier coefficients. The new Fourier coefficients determine a new solution.
}

struction, at least on shell. Since we are dealing with two copies of the same system, all that was said above is true for the tilde string. However, note that at finite temperature the notions of string at tilde string are slightly different since both of them mix the operators of string and tilde string as they were defined initially at $T=0$. In this sense, the tilde symbol just reminds us that the operators were obtained from the copy of the original system.

\section{B. Boundary conditions at $T \neq 0$}

To define the $\mathrm{D} p$-branes at $T \neq 0$, we have to construct the counterpart of the boundary conditions (1) at finite temperature for both, the string and the tilde string. We have three possibilities to do that: first, to map the operators that describe the relations (1) at finite $T \neq 0$, second, to map the operators and the states, or third, to map the states at finite temperature while keeping the operators at $T=0$. The first two alternatives give the solutions at finite temperature. The last one gives no new information since its solutions should be among the solutions at zero temperature. This reminds us of the different pictures in quantum mechanics, and it would be interesting to see the relation between all these representations. In what follows we will map the operators at $T=0$ using the Bogoliubov transformation; it will turn out that the states that satisfy these boundary conditions depend explicitly on $T$ [56]. It is possible to formally derive the boundary conditions from an action. Since the properties of the bosonic string are satisfied, the string equations can be obtained from a formal string action, which mimics the zerotemperature action and depends implicitly on the parameter $\beta_{T}$. Its form is given by the following relation:

$$
\begin{aligned}
S\left(\beta_{T}\right)= & \frac{1}{4 \pi \alpha^{\prime}} \int d \tau \int d \sigma\left\{\partial^{\alpha} X^{\mu}\left(\beta_{T}\right) \partial_{\alpha} X_{\mu}\left(\beta_{T}\right)\right. \\
& \left.-\left\{\delta(\sigma) \dot{X}^{\mu}\left(\beta_{T}\right) A_{\mu}\left[X\left(\beta_{T}\right)\right]\right\}\right\},
\end{aligned}
$$

which is the action of a open string that couples with a $U(1)$ field on a $\mathrm{D} p$-brane located at $\sigma=0$ on the world sheet. We assume that the gauge field is nonzero along the directions parallel to the world volume of the brane and $A^{i}\left[X\left(\beta_{T}\right)\right]$ $=$ const. Moreover, we do not consider any explicit dependence on the temperature of the gauge field. Then the boundary conditions in the closed string sector take the usual form

$$
\begin{aligned}
{\left.\left[\partial_{\tau} X_{a}\left(\beta_{T}\right)+F_{b a} \partial_{\sigma} X^{b}\left(\beta_{T}\right)\right]\right|_{\tau=0} } & =0, \\
X^{i}\left(\beta_{T}\right)-\left.y^{i}\right|_{\tau=0} & =0 .
\end{aligned}
$$

Since the solutions of the equations of motion that enter Eq. (34) have the form of a closed string solution with the oscillator operators replaced by the operators at $T \neq 0$, one can express Eq. (34) in terms of creation and annihilation operators 


$$
\begin{array}{r}
(\mathbb{I}+\mathbb{F}){ }_{b}^{a} A_{n}^{b}\left(\beta_{T}\right)+(\mathbb{I}-\mathbb{F})_{b}^{a} B_{n}^{b \dagger}\left(\beta_{T}\right)=0, \\
(\mathbb{I}+\mathbb{F}){ }_{b}^{a} A_{n}^{b \dagger}\left(\beta_{T}\right)+(\mathbb{I}-\mathbb{F}){ }_{b}^{a} B_{n}^{b}\left(\beta_{T}\right)=0, \\
A_{n}^{i}\left(\beta_{T}\right)-B_{n}^{i \dagger}\left(\beta_{T}\right)=0, \\
A_{n}^{i \dagger}\left(\beta_{T}\right)-B_{n}^{i}\left(\beta_{T}\right)=0,
\end{array}
$$

for any $n>0$, which are supplemented by the boundary conditions for the position of the center-of-mass of string and its conjugate momenta

$$
\begin{aligned}
p^{a} & =0, \\
q^{i}-y^{i} & =0 .
\end{aligned}
$$

The last two relations are a consequence of the fact that the operators $\hat{p}, \hat{X}, \hat{\tilde{p}}$, and $\hat{\tilde{X}}$ commute with all oscillator operators. Therefore, they are not affected by the Bogoliubov transformations. If we construct the zero mode $G$ operators, as we did for the oscillators, we see that the momenta commute with them. Thus, we can take the position and momenta operators of both, the string and the tilde string, to be invariant under the transformations above. Also the corresponding eigenstates of the momenta operators are taken to be invariant.

Some comments are in order now. We note that the above construction should be applied to the tilde string as well. Thus, we will have two boundary conditions, where the second one is identical to Eq. (35) with all the operators replaced by the corresponding tilde operators. These boundary conditions can be constructed with the same mapping or from a formal action of the form (32). It is not possible to construct the boundary conditions from a Lagrangian at finite temperature since that is not the basic object in thermo field dynamics, which only deals with a Lagrangian that depends on the fields at $T=0$ of the form $\mathcal{L}-\widetilde{\mathcal{L}}$, where the first term contains information about the string and the second term is the Lagrangian of the tilde string [51]. Then the boundary conditions should be imposed as we have already commented at the beginning of this paragraph, i.e., by mapping them to finite temperature. We could have started from the thermo field Lagrangian to obtain the boundary conditions at $T=0$, which are just our conditions since the two terms in the thermo field Lagrangian are independent, and then map each of them at $T \neq 0$. Therefore, solving the boundary conditions within the thermo field framework is equivalent to solving them directly from the Lagrangian (32) and its copy.

\section{Boundary states at $T \neq 0$}

Our next goal is to solve the equations (35) and the corresponding ones for the tilde fields. To this end, we note that Eq. (35) has the same form as Eq. (1). Moreover, the operators at $T \neq 0$ and the thermal vacuum have the same properties as the ones at $T=0$. Therefore, the solutions to the boundary conditions (35) are given by

$$
\begin{aligned}
\left.\left|B\left(\beta_{T}\right)\right\rangle\right\rangle= & N_{p}\left(F, \beta_{T}\right) \delta^{\left(d_{\perp}\right)}(\hat{q}-y) \\
& \left.\times \exp \left[-\sum_{n=1}^{\infty} A^{\mu \dagger}\left(\beta_{T}\right) M_{\mu \nu} B^{\nu \dagger}\left(\beta_{T}\right)\right]\left|0\left(\beta_{T}\right)\right\rangle\right\rangle,
\end{aligned}
$$

where $N_{p}\left(F, \beta_{T}\right)$ is the normalization constant at $T \neq 0$. An eigenstate of the momentum of the center-of-mass is included in the thermal vacuum. In a similar manner one can write the solution to the other boundary condition for the tilde string. The total solution will be a product of them, and it is given by the following formula:

$$
\begin{aligned}
\left.\left|B\left(\beta_{T}\right)\right\rangle\right\rangle_{1}= & N_{p}^{2}\left(F, \beta_{T}\right) \delta^{\left(d_{\perp}\right)}(\hat{q}-y) \delta^{\left(d_{\perp}\right)}(\tilde{\tilde{q}}-\tilde{y}) \\
& \times e\left[-\sum_{n=1}^{\infty} A_{n}^{\dagger}\left(\beta_{T}\right) M B_{n}^{\dagger}\left(\beta_{T}\right)\right] \\
& \left.\times \exp \left[-\sum_{n=1}^{\infty} \widetilde{A}_{n}^{\dagger}\left(\beta_{T}\right) M \widetilde{B}_{n}^{\dagger}\left(\beta_{T}\right)\right]\left|0\left(\beta_{T}\right)\right\rangle\right\rangle .
\end{aligned}
$$

Here, the normalization constants for the two solutions were taken to be equal because we deal with identical copies of the system. To compute it, we should calculate the scattering amplitude in the closed string channel and in the open string channel and compare the results. However, since the solutions are formally identical to the ones at $T=0$, one can set $N_{p}\left(F, \beta_{T}\right)=N_{p}(F)$ which is known to be proportional to the Born-Infeld action $[49,50]$

$$
N_{p}\left(F, \beta_{T}\right)=N_{p}(F)=\sqrt{-\operatorname{det}(\delta+\hat{F})} .
$$

The solution (39) was obtained using just the algebra of the operators $A_{\beta_{T}}, B_{\beta_{T}}, \widetilde{A}_{\beta_{T}}$, and $\widetilde{B}_{\beta_{T}}$ and the $G$ operators. Since their action on the vacuum is similar to that at $T=0$, Eq. (39) has formally the same form as the boundary state at zero temperature (4). The difference is that the new solutions contain an explicit dependence on the temperature.

Another solution can be obtained observing that the boundary condition (35) can be written as

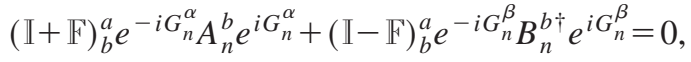

$$
\begin{aligned}
& (\mathbb{I}+\mathbb{F})_{b}^{a} e^{-i G_{n}^{\alpha} A_{n}^{\dagger b}} e^{i G_{n}^{\alpha}}+(\mathbb{I}-\mathbb{F})_{b}^{a} e^{-i G_{n}^{\beta}} B_{n}^{b} e^{i G_{n}^{\beta}}=0,
\end{aligned}
$$

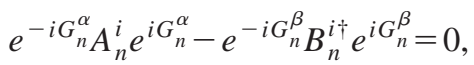

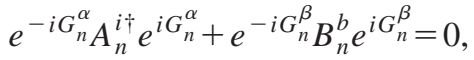

for any $n>0$. Similar equations can be written for the boundary of the tilde string with tilde operators instead. Then one can see that there is a solution to the equations (41) of the form

$$
\left.\left|B\left(\beta_{T}\right)\right\rangle\right\rangle_{2}=N_{p}\left(\beta_{T}\right) \prod_{n=1}^{\infty} e^{-i G_{n}^{\alpha}} \prod_{m=1}^{\infty} e^{-i G_{m}^{\beta}}|B\rangle,
$$


where $|B\rangle$ is the boundary state at $T=0$ given in Eq. (4). A similar expression can be found for the tilde operators and the second solution to the boundary conditions at $T \neq 0$ has the form

$$
\left.\left|B\left(\beta_{T}\right)\right\rangle\right\rangle_{2}=|B\rangle \widetilde{\left|B\left(\beta_{T}\right)\right\rangle} .
$$

Note that the solutions (39) and (43) are different because the exponential contains different operators. They have a natural degeneracy due to the fact that we work in a doubled Fock space and they are physical solutions in the light-cone gauge. In a conformal gauge, the contributions of ghosts at finite temperature [55] should be taken into account in order to eliminate the unphysical degrees of freedom of closed string. In what follows, we study the solution (39). Solution (43) represents the mapping of the boundary state at $T=0$ at finite temperature via Bogoliubov operators.

\section{ENTROPY OF D-BRANES}

It is interesting to investigate now the thermal properties of the boundary states obtained in the previous section. Since we are working with closed string, we are going to compute its entropy in the boundary state (39).

According to the thermo field dynamics [51], the entropy operators for the bosonic closed string in terms of string oscillators and in $k_{B}$ units, are given by the following relations:

$$
\begin{aligned}
K= & \sum_{\mu} \sum_{n}\left[\left(A_{n}^{\mu \dagger} A_{n}^{\mu}+B_{n}^{\mu \dagger} B_{n}^{\mu}\right) \log \sinh ^{2} \theta_{n}\right. \\
& \left.-\left(A_{n}^{\mu} A_{n}^{\mu \dagger}+B_{n}^{\mu} B_{n}^{\mu \dagger}\right) \log \cosh ^{2} \theta_{n}\right],
\end{aligned}
$$

for the string, and

$$
\begin{aligned}
\widetilde{K}= & \sum_{\mu} \sum_{n}\left[\left(\widetilde{A}_{n}^{\mu \dagger} \widetilde{A}_{n}^{\mu}+\widetilde{B}_{n}^{\mu \dagger} \widetilde{B}_{n}^{\mu}\right) \log \sinh ^{2} \theta_{n}\right. \\
& \left.-\left(\widetilde{A}_{n}^{\mu} \widetilde{A}_{n}^{\mu \dagger}+\widetilde{B}_{n}^{\mu} \widetilde{B}_{n}^{\mu \dagger}\right) \log \cosh ^{2} \theta_{n}\right]
\end{aligned}
$$

for the tilde string, respectively. Using the $G$-operators algebra, it is easy to show that

$$
\left[K-\widetilde{K}, G^{\alpha}\right]=0, \quad\left[K-\widetilde{K}, G^{\beta}\right]=0,
$$

where the operators $G^{\alpha}$ and $G^{\beta}$ are defined as

$$
G^{\alpha}=\sum_{n} G_{n}^{\alpha}, \quad G^{\beta}=\sum_{n} G_{n}^{\beta}
$$

In order to compute the entropy, one has to find the expectation value of the operator (44) in the state (39). In the thermo field dynamics it is postulated that the physical properties of the system should be given in terms of operators without the tilde [51]. Therefore, we do not compute the expectation value of the $\widetilde{K}$ operator.

It is useful to write the expectation value of the entropy operator in the following form:

$$
\begin{aligned}
{ }_{1}\left\langle\left\langle B\left(\beta_{T}\right)|K| B\left(\beta_{T}\right)\right\rangle\right\rangle_{1} \\
={ }_{1}\left\langle\left\langle 0\left(\beta_{T}\right)\left|e^{F^{\dagger}} K e^{F}\right| 0\left(\beta_{T}\right)\right\rangle\right\rangle_{1} \\
={ }_{1}\left\langle\left\langle 0\left(\beta_{T}\right)\left|e^{F^{\dagger}} e^{F} C\right| 0\left(\beta_{T}\right)\right\rangle\right\rangle_{1} \\
\quad+{ }_{1}\left\langle\left\langle 0\left(\beta_{T}\right)\left|e^{F^{\dagger}} e^{F} K\right| 0\left(\beta_{T}\right)\right\rangle\right\rangle_{1},
\end{aligned}
$$

where we are using the notation

$$
F=F\left(\beta_{T}\right)=\exp \left[-\sum_{n=1}^{\infty} A_{n}^{\dagger}\left(\beta_{T}\right) M B_{n}^{\dagger}\left(\beta_{T}\right)\right]
$$

and

$$
C=[K, F] .
$$

To compute the terms in Eq. (48) we should work with the operators defined either at finite temperature or at zero temperature, since the action of these operators on the corresponding vacua is known. The two ways of doing computations are completely equivalent. The following relations are useful:

$$
\begin{gathered}
\left.u_{m}^{2} A_{m}^{\rho^{\dagger}}\left(\beta_{T}\right) A_{m}^{\rho}(\beta)\left|0\left(\beta_{T}\right)\right\rangle\right\rangle=0, \\
\left.\left.u_{m} v_{m} A_{m}^{\rho^{\dagger}}\left(\beta_{T}\right) \widetilde{A}_{m}^{\rho \dagger}\left(\beta_{T}\right)\left|0\left(\beta_{T}\right)\right\rangle\right\rangle=u_{m} v_{m}\left|\left(1_{m}\right)^{\rho \tilde{\rho}} ; 0 ; \beta_{T}\right\rangle\right\rangle, \\
\left.u_{m} v_{m} \widetilde{A}_{m}^{\rho}\left(\beta_{T}\right) A_{m}^{\rho}\left(\beta_{T}\right)\left|0\left(\beta_{T}\right)\right\rangle\right\rangle=0 \\
\left.\left.v_{m}^{2} \widetilde{A}_{m}^{\rho}\left(\beta_{T}\right) \widetilde{A}_{m}^{\rho^{\dagger}}\left(\beta_{T}\right)|0(\beta)\rangle\right\rangle=v_{m}^{2}\left|0\left(\beta_{T}\right)\right\rangle\right\rangle
\end{gathered}
$$

where we have used the shorthand notation

$$
\left.\left.\left.\left|\left(1_{m}\right)^{\rho \tilde{\rho}} ; 0 ; \beta_{T}\right\rangle\right\rangle=\left|1, \tilde{1} ; m, \tilde{m} ; \beta_{T}\right\rangle\right\rangle_{\alpha}^{\rho \tilde{\rho}}\left|0\left(\beta_{T}\right)\right\rangle\right\rangle_{\beta},
$$

and

$$
\begin{gathered}
\left.\left|1, \tilde{1} ; m, \tilde{m} ; \beta_{T} h t\right\rangle\right\rangle_{\alpha}^{\rho \tilde{\rho}} \\
=\mid 0, \ldots, 1, \ldots, 0 ; 0, \ldots, \tilde{1}, \ldots, 0 ; \ldots, m, \ldots ; \\
\left.\left.\quad \ldots, \tilde{m}, \ldots ; \beta_{T}\right\rangle\right\rangle_{\alpha}^{\rho \tilde{\rho}} .
\end{gathered}
$$

Here, the indices $\rho$ and $\tilde{\rho}$ indicate that the state has one quanta in the $X^{\rho}$ direction of space time. The space time is the same for the string as well as for the tilde string since doubling the system does not mean doubling the space time. The tilde over the index just indicates that there is a state, for the tilde string in that direction, that should be taken into account. The first 1 on the right-hand side of Eq. (53) means that the quanta is of string type, in the $\rho$ th direction. Zero stands for the other directions. The $\widetilde{1}$ means that one quanta of tilde string exists in that state and in the same direction $\rho$ and there are no other quanta in the other directions of space time. $m$ is the mode of the string quanta, while $\tilde{m}$ is the mode of the tilde-string quanta. The modes that are on the rightmoving sectors of the closed string and tilde string are denoted by the index $\alpha$. Since the oscillators are independent in all directions and for all different modes, the orthogonality 
relations among these states are easy to write down. We also assume that the states are orthonormal.

Using the properties of $G$ operators and the relations (51) we can write the action of the entropy operator on the boundary state at finite temperature under the form

$$
\begin{aligned}
\left.K e^{F}\left|0\left(\beta_{T}\right)\right\rangle\right\rangle_{1} & \\
= & -\sum_{\mu, \nu=1}^{24} \sum_{k=1}^{\infty} \frac{(-)^{k}}{k !}\left[A_{n}^{\mu \dagger}\left(\beta_{T}\right) M^{\mu \nu} B_{n}^{\nu^{\dagger}}\left(\beta_{T}\right)\right]^{k} \\
& \times \sum_{m=1}^{\infty}\left\{\log \tanh ^{2} \theta_{m} \sum_{\rho=1}^{24}\left[u_{m} v_{m}\left|\left(1_{m}\right)^{\rho} ; 0 ; \beta_{T}\right\rangle\right\rangle\right] \\
& \left.\left.+48\left[v_{m}^{2} \log \tanh ^{2} \theta_{m}-\log \cosh ^{2} \theta_{m}\right]\left|0\left(\beta_{T}\right)\right\rangle\right\rangle\right\} .
\end{aligned}
$$

The first term can be written in terms of the states (53) as

$$
\begin{aligned}
\sum_{k=1}^{\infty} \frac{(-)^{k}}{k !}\left[A_{n}^{\mu \dagger}\left(\beta_{T}\right)\right]^{k}\left[M_{\mu \nu}\right]^{k}\left[B_{n}^{\nu^{\dagger}}\left(\beta_{T}\right)\right]^{k} \\
\left.\quad \times(-) \sum_{m=1}^{\infty}\left\{\log \tanh ^{2} \theta_{m} \sum_{\rho=1}^{24}\left[u_{m} v_{m}\left|\left(1_{m}\right)^{\rho} ; 0 ; \beta_{T}\right\rangle\right\rangle\right]\right\} \\
=\mid 0, \ldots, 1, \ldots, k, \ldots, 0 ; 0, \ldots, \tilde{1}, \ldots, 0 \\
\left.\left.\quad \ldots, m, \ldots, n, \ldots ; \ldots, \tilde{m}, \ldots ; \beta_{T}\right\rangle\right\rangle_{\alpha}^{\rho \tilde{\rho} \mu} \\
\otimes \mid 0, \ldots, 0, \ldots, k, \ldots, 0 ; 0, \ldots, 0, \ldots, 0 \\
\left.\left.\quad \ldots, n, \ldots ; \ldots ; \beta_{T}\right\rangle\right\rangle_{\beta}^{\nu}
\end{aligned}
$$

The relations (54) and (55) are important for computing the second term in Eq. (48) while the first term can be shown to be of the following form:

$$
\begin{aligned}
{ }_{1}\left\langle\left\langle 0\left(\beta_{T}\right)\left|e^{F^{\dagger}} e^{F} C\right| 0\left(\beta_{T}\right)\right\rangle\right\rangle_{1} \\
=2 \prod_{m=1}^{\infty} \prod_{\mu=1}^{24} \prod_{\nu=1}^{24} \sum_{k=0}^{\infty} u_{m}^{2}\left(\beta_{T}\right) \frac{(-)^{2 k+1} M_{\mu \nu}^{2 k+2}}{k !(k+1) !}
\end{aligned}
$$

After some tedious algebra one obtains from Eqs. (55) and (56) the following value for the entropy of the closed string in the boundary state at finite temperature:

$$
\begin{aligned}
K_{D p}= & { }_{1}\left\langle\left\langle B\left(\beta_{T}\right)|K| B\left(\beta_{T}\right)\right\rangle\right\rangle_{1} \\
= & 48 \sum_{m=1}^{\infty}\left[\log \sinh ^{2} \theta_{m}-\sinh ^{2} \theta_{m} \log \tanh ^{2} \theta_{m}\right] \\
& +2 \prod_{m=1}^{\infty} \prod_{\mu=1}^{24} \prod_{\nu=1}^{24} \sum_{k=0}^{\infty} \cosh ^{2} \theta_{m} \frac{(-)^{2 k+2} M_{\mu \nu}^{2 k+2}}{k !(k+1) !} .
\end{aligned}
$$

The first two terms obtained above do not depend on the gauge field $F_{a b}$. It is easy to see that in the $T \rightarrow 0$ limit the contribution of the oscillators to $K_{D p}$ diverges. If we subtract the infinity we are left with the last term that may converge but towards a positive number only. However, in the $T \rightarrow \infty$, the contribution of the oscillator behaves as $\log (-1)$. This might be an indication that the notion of temperature breaks down for arbitrary large temperature due to a similar phenomenon that occurs in string theory at Hagedorn temperature. We do not have a good explanation for this behavior.

\section{SUMMARY AND DISCUSSIONS}

In this paper we constructed the bosonic boundary states at finite temperature, which were obtained by solving the boundary conditions of the bosonic closed string. The boundary conditions at $T \neq 0$ were obtained by mapping the corresponding boundary conditions at $T=0$ with thermal Bogoliubov transformations. Since the states at $T=0$ have the interpretation of $\mathrm{D} p$-branes, we interpret the solutions we have found as $\mathrm{D} p$-branes at finite temperature. The construction was done in the framework of the thermo field dynamics [51]. We reproduced the states obtained in a previous work [56] given by Eq. (39) and corrected some misprints in that paper and also, we obtained a new solution (43), which represents the Bogoliubov map of the $\mathrm{D} p$-branes at zero temperature.

The crucial point in our construction was to map the boundary conditions at $T \neq 0$. We have adopted the point of view that the dependence on the temperature should be encoded in the operators, which are in agreement with thermo field dynamics. In this way we have obtained two sets of boundary conditions, one for the original string and another one for its copy. In Ref. [56] another solution of one set of boundary conditions was discussed. Since the total Fock space is a direct product of two copies of the Fock space of a bosonic closed string, a linear combinations of boundary states described by the following operators can be given ${ }^{2}$

$$
\begin{aligned}
\hat{O}_{2,3}= & N_{p}\left(F, \beta_{T}\right)\left(\prod_{n=1}^{\infty} e^{-A_{n}^{\dagger}\left(\beta_{T}\right) M B_{n}^{\dagger}\left(\beta_{T}\right)} \times 1 \pm 1\right. \\
& \left.\times \prod_{n=1}^{\infty} e^{-\widetilde{A}_{n}^{\dagger}\left(\beta_{T}\right) M \tilde{B}_{n}^{\dagger}\left(\beta_{T}\right)}\right)
\end{aligned}
$$

where an implicit delta function that localizes the center-ofmass of the string and the tilde string are assumed to exist. The operators (58) act on the thermal vacuum. We note that the vectors constructed with Eq. (58) satisfy one set of boundary conditions, say, for the string, but do not satisfy the boundary conditions for the tilde string.

For the $\mathrm{D} p$-brane (39) which has the same form as the $\mathrm{D} p$-brane at $T=0$, the entropy was given in Eq. (57). We found that in the limit $T \rightarrow 0$, the contribution of the oscillators diverges. If this is subtracted from the total value of the entropy, what is left is a series of positive terms with a slower increment than the exponential function. When the limit $T \rightarrow \infty$ is taken, the oscillator contribution breaks down since it involves the logarithm of negative unity. One possible explanation is that using bosonic strings is inappro-

\footnotetext{
${ }^{2}$ These solutions were obtained in Ref. [56]. There the operators should be read at $T \neq 0$.
} 
priate for describing $\mathrm{D} p$-branes at high temperatures since the phase transition of string theory around the Hagedorn temperature makes the statistics of strings unfeasible [3], and consequently the boundary states. The tachyons, the gravitons, and the dilaton are responsible for this phenomenon. In the present case only physical modes have been considered since we have worked in the light-cone gauge. Nevertheless, the contributions of the massless modes can induce a bad behavior for the entropy. It is worthwhile to investigate the contributions that can be obtained from the fermionic part in the case of superbranes. Another possibility would be that $\mathrm{D} p$-branes at finite temperature are not acceptable boundary states for closed string theory at $T \neq 0$. However, to give a definitive answer to these problems, further investigations of the thermal properties of the boundary states should be performed. We hope to report on these topics in a future work [57].

\section{ACKNOWLEDGMENTS}

We would like to thank N. Berkovits, P. K. Panda, B. M. Pimentel, H. Q. Placido, D. L. Nedel, and B. C. Vallilo for useful discussions. I. V. V. also acknowledges M. A. De Andrade and J. A. Helayel-Neto for hospitality at GFT-UCP, where part of this work was done. This work was supported by FAPESP (I.V.V.) and CAPES (A.L.G.).

\section{APPENDIX A}

In this appendix we review some basic relations of thermo field theory with focus in the bosonic harmonic oscillator. We closely follow Ref. [51].

The basic idea of thermo field dynamics is to construct statistical averages of some operator $A$ in terms of expectation values in a vacuum that presents a dependence with the temperature:

$$
\langle A\rangle \equiv Z^{-1}\left(\beta_{T}\right) \operatorname{Tr}\left[e^{-\beta_{T}^{H}} A\right]=\left\langle\left\langle 0\left(\beta_{T}\right)|A| 0\left(\beta_{T}\right)\right\rangle\right\rangle,
$$

where $\beta_{T}=\left(k_{B} T\right)^{-1}$ is the Botzmann's constant and

$$
H|n\rangle=E_{n}|n\rangle .
$$

The eigenstates of the Hamiltonian $H$ of the system are supposed to be orthonormal. The partition function is defined by the usual relation

$$
Z\left(\beta_{T}\right)=\operatorname{Tr}\left[e^{-\beta_{T} H}\right] .
$$

It is possible to realize such of idea if a duplication of the physical Hilbert space is performed, i.e., if one considers the Hilbert space as a direct product of two spaces: the original one and a nonphysical copy of it. The nonphysical or auxiliary subspace is represented by a tilde and it is acted on by a copy of the operators of the original system, e.g.,

$$
\tilde{H}|\tilde{n}\rangle=E_{n}|\tilde{n}\rangle \text {. }
$$

As a consequence any state in the total Hilbert space has the following form:

$$
|n, \tilde{m}\rangle=|n\rangle \otimes|\tilde{m}\rangle
$$

The temperature dependent vacuum can be written as

$$
\left.\left|0\left(\beta_{T}\right)\right\rangle\right\rangle=Z^{-1 / 2}\left(\beta_{T}\right) \sum_{n} e^{-(1 / 2) \beta_{T} E_{n}}|n, \tilde{m}\rangle,
$$

and it is called "thermal vacuum."

One simple system for which the above construction is straightforward is the single bosonic oscillator

$$
H=\omega a^{\dagger} a
$$

with the known algebra

$$
\left[a^{\dagger}, a\right]=1 ; \quad[a, a]=\left[a^{\dagger}, a^{\dagger}\right]=0,
$$

and the Fock space spanned by the following states

$$
|n\rangle=\frac{\left(a^{\dagger}\right)^{n}}{\sqrt{n !}}|0\rangle
$$

for all positive integers $n$. The tilde oscillator has identical properties

$$
\widetilde{H}=\omega \tilde{a}^{\dagger} \tilde{a}
$$

and

$$
\left[\tilde{a}^{\dagger}, \tilde{a}\right]=1 ; \quad[\tilde{a}, \tilde{a}]=\left[\tilde{a}^{\dagger}, \tilde{a}^{\dagger}\right]=0 .
$$

It is independent of the first oscillator, i.e., the two Hilbert spaces are completely orthogonal:

$$
[a, \tilde{a}]=\left[a, \tilde{a}^{\dagger}\right]=\left[a^{\dagger}, \tilde{a}^{\dagger}\right]=\left[a^{\dagger}, \tilde{a}\right]=0 .
$$

The duplicated vacuum is defined by

$$
a|0\rangle\rangle=\tilde{a}|0\rangle\rangle
$$

which has a solution in terms of the two vacua

$$
|0\rangle\rangle \equiv|0, \widetilde{0}\rangle=|0\rangle \otimes \widehat{0\rangle}
$$

The Hamiltonian of the total system is defined by the following relation:

$$
\hat{H}=H-\tilde{H}
$$

A simple algebra shows that the thermal vacuum can be written in terms of a coherent state

$$
\left.\left.\left|0\left(\beta_{T}\right)\right\rangle\right\rangle=\frac{1}{u\left(\beta_{T}\right)} \exp \left[\frac{v\left(\beta_{T}\right)}{u\left(\beta_{T}\right)} a^{\dagger} \tilde{a}^{\dagger}\right]|0\rangle\right\rangle .
$$

The partition function of the oscillator is given by

$$
Z\left(\beta_{T}\right)=\frac{1}{1-e^{-\beta_{T} \omega}},
$$


ensuring that the thermal vacuum has unit norm. Also, the temperature-dependent coefficients are defined by the relations

$$
\begin{aligned}
& u\left(\beta_{T}\right) \equiv\left(1-e^{-\beta_{T^{\omega}}}\right)^{-1 / 2}=\cosh \theta\left(\beta_{T}\right), \\
& v\left(\beta_{T}\right) \equiv\left(e^{\beta_{T^{\omega}}}-1\right)^{-1 / 2}=\sinh \theta\left(\beta_{T}\right) .
\end{aligned}
$$

If we consider unitary Bogoliubov operators having the form

$$
G_{B} \equiv-i \theta\left(\beta_{T}\right)\left(a \tilde{a}-a^{\dagger} \tilde{a}^{\dagger}\right),
$$

it is easy to show that the thermal vacuum is obtained from the total vacuum at $T=0$ by a simple Bogoliubov transformation

$$
\left.\left.\left|0\left(\beta_{T}\right)\right\rangle\right\rangle=e^{-i G_{B}}|0\rangle\right\rangle .
$$

The operators at finite temperature are also generated by the Bogoliubov operators

$$
\begin{array}{ll}
a\left(\beta_{T}\right)=e^{-i G_{b}} a & e^{i G_{b}}=u\left(\beta_{T}\right) a-v\left(\beta_{T}\right) \tilde{a}^{\dagger}, \\
\tilde{a}\left(\beta_{T}\right)=e^{-i G_{b}} \tilde{a} & e^{i G_{b}}=u\left(\beta_{T}\right) \tilde{a}-v\left(\beta_{T}\right) a^{\dagger},
\end{array}
$$

and they act on the thermal vacuum (justifying its name) as

$$
\left.\left.a\left(\beta_{T}\right)\left|0\left(\beta_{T}\right)\right\rangle\right\rangle=\tilde{a}\left(\beta_{T}\right)\left|0\left(\beta_{T}\right)\right\rangle\right\rangle=0 .
$$

With this construction, the Fock space is spanned by the vectors

$$
\left.\left.\left|0\left(\beta_{T}\right)\right\rangle\right\rangle, \quad a^{\dagger}\left(\beta_{T}\left|0\left(\beta_{T}\right)\right\rangle\right\rangle, \quad \tilde{a}^{\dagger}\left(\beta_{T}\right)\left|0\left(\beta_{T}\right)\right\rangle\right\rangle, \ldots
$$

$$
\left.\frac{1}{\sqrt{n !}} \frac{1}{\sqrt{m !}}\left[a^{\dagger}\left(\beta_{T}\right)\right]^{n}\left[\tilde{a}^{\dagger}\left(\beta_{T}\right)\right]^{m}\left|0\left(\beta_{T}\right)\right\rangle\right\rangle, \ldots
$$

The commutation relation between the temperaturedependent operators are the same as for the duplicated system at zero temperature. It is easy to show that the $G$ operator is a constant of motion:

$$
[G, \hat{H}]=[G, H-\widetilde{H}]=0 .
$$

The construction presented above can be straightforward extended to a set of infinite free bosonic oscillators. In that case the Bogoliubov operator is given by

$$
G_{B} \equiv-i \sum_{n} \theta\left(\beta_{T}\right)\left(a_{n} \tilde{a}_{n}-a_{n}^{\dagger} \tilde{a}_{n}^{\dagger}\right)
$$

and the thermal vacuum can be expressed as

$$
\left.\left.\left|0\left(\beta_{T}\right)\right\rangle\right\rangle=e^{-1 / 2 K} \exp \left\{\sum_{n} a_{n}^{\dagger} \tilde{a}_{n}^{\dagger}\right\}|0\rangle\right\rangle,
$$

where

$$
K=-\sum_{n}\left\{a_{n}^{\dagger} a_{n} \log \sinh ^{2} \theta_{n}-a_{n} a_{n}^{\dagger} \log \cosh ^{2} \theta_{n}\right\} .
$$

$K$ is called the entropy operator. This interpretation comes from the fact that the vacuum expectation value of $K$ times the Boltzmann constant $k_{B}$ is the entropy of the physical system.
[1] For a review see L. Alvarez-Gaumé and M.A. Vázquez-Mozo, Topics in String Theory and Quantum Gravity, Les Houches, Session LVII, 1992, edited by B. Julia and J. Zinn-Justin.

[2] D.J. Gross and P. Mende, Nucl. Phys. B307, 407 (1988); D.J. Gross, Phys. Rev. Lett. 60, 1229 (1988).

[3] J.J. Atick and E. Witten, Nucl. Phys. B310, 291 (1988).

[4] E. Alvarez, Phys. Rev. D 31, 418 (1985); Nucl. Phys. B269, 596 (1986).

[5] E. Alvarez and M. Osorio, Phys. Rev. D 36, 1175 (1987).

[6] Y. Leblanc, Phys. Rev. D 38, 3087 (1988).

[7] R. Brandenberger and C. Vafa, Nucl. Phys. B316, 391 (1989).

[8] J. Kipfganz and H. Perlt, Class. Quantum Grav. 5, 453 (1988).

[9] A.K. Chaudhuri, hep-th/9706104.

[10] Y. Leblanc, Phys. Rev. D 36, 1780 (1987); 37, 1547 (1988); 39, 1139 (1989); 39, 3731 (1989).

[11] Y. Leblanc, M. Knecht, and J.C. Wallet, Phys. Lett. B 237, 357 (1990)

[12] E. Ahmed, Int. J. Theor. Phys. 26, 1135 (1987); Phys. Rev. Lett. 60, 684 (1988).

[13] J. Polchinski, Phys. Rev. Lett. 75, 4724 (1995).

[14] J. Polchinski, String Theory (Cambridge University, Cambridge, England, 1999).

[15] C. Johnson, D-brane Primer, hep-th/0007170.
[16] A. Sen, Nucl. Phys. B440, 421 (1995).

[17] A. Strominger and C. Vafa, Phys. Lett. B 379, 99 (1996).

[18] L. Susskind, hep-th/9309145.

[19] G. Horowitz and J. Polchinski, Phys. Rev. D 55, 6189 (1997).

[20] T. Banks, W. Fischler, I. Klebanov, and L. Susskind, Phys. Rev. Lett. 80, 226 (1998); J. High Energy Phys. 01, 008 (1998).

[21] S. Chaudhuri and D. Minic, Phys. Lett. B 433, 301 (1998).

[22] I. Klebanov and L. Susskind, Phys. Lett. B 416, 62 (1998).

[23] E. Halyo, hep-th/9709225.

[24] S. Das, S. Mathur, S. Kalyana Rama, and P. Ramadevi, Nucl. Phys. B527, 187 (1998).

[25] G. Horowitz and E. Martinec, Phys. Rev. D 57, 4935 (1998).

[26] M. Li, J. High Energy Phys. 01, 009 (1998); M. Li and E. Martinec, hep-th/9801070.

[27] T. Banks, W. Fischler, and I. Klebanov, Phys. Lett. B 423, 54 (1998).

[28] H. Liu and A. Tseytlin, J. High Energy Phys. 01, 010 (1998).

[29] D. Minic, hep-th/9712202.

[30] A. Strominger, Phys. Rev. Lett. 71, 3397 (1993).

[31] I.V. Volovich, hep-th/9608137.

[32] J. Maldacena and A. Strominger, J. High Energy Phys. 07, 013 (1998). 
[33] M.V. Mozo, Phys. Lett. B 388, 494 (1996); J.L.F. Barbon and M.V. Mozo, Nucl. Phys. B497, 236 (1997).

[34] A. Bytsenko, S. Odintsov, and L. Granada, Mod. Phys. Lett. A 11, 2525 (1996).

[35] J. Ambjorn, Yu. Makeenko, G.W. Semenoff, and R. Szabo, Phys. Rev. D 60, 106009 (1999).

[36] G. Dvali, I.I. Kogan and M. Shifman, Phys. Rev. D 62, 106001 (2000).

[37] S. Abel, K. Freese, and I.I. Kogan, J. High Energy Phys. 01, 039 (2001).

[38] I.I. Kogan, A. Kovner, and M. Schvellinger, hep-th/0103235.

[39] S.A. Abel, J.L.F. Barbon, I.I. Kogan, and E. Rabinovici, J. High Energy Phys. 04, 015 (1999).

[40] J.L.F. Barbon and E. Rabinovici, J. High Energy Phys. 06, 029 (2001).

[41] S.S. Gubser, I.R. Klebanov, M. Rangamani, and E. Witten, hep-th/0009140.

[42] C.G. Callan, C. Lovelace, C.R. Nappi, and S.A. Yost, Nucl. Phys. B288, 525 (1987); B293, 23 (1987); B308, 221 (1988).

[43] J. Polchinski and Y. Cai, Nucl. Phys. B296, 91 (1988).
[44] M.B. Green and P. Wai, Nucl. Phys. B431, 131 (1994).

[45] M.B. Green, Nucl. Phys. B381, 201 (1992).

[46] M.B. Green and M. Gutperle, Nucl. Phys. B476, 484 (1996).

[47] M. Li, Nucl. Phys. B460, 351 (1996).

[48] C. Callan Jr. and I. Klebanov, Nucl. Phys. B465, 473 (1996).

[49] P. Di Vecchia and A. Liccardo, D-Branes in String Theory, hep-th/9912161; hep-th/9912275.

[50] P. Di Vecchia, M. Frau, A. Lerda, and A. Liccardo, Nucl. Phys. B565, 397 (2000).

[51] Y. Takahashi and H. Umezava, Collect. Phenom. 2, 55 (1975).

[52] H. Fujisaki and K. Nakagawa, Prog. Theor. Phys. 82, 236 (1989); 82, 1017 (1989); 83, 18 (1990); Europhys. Lett. 20, 677 (1992); 28, 471 (1994).

[53] H. Fujisaki, Nuovo Cimento A 108, 1079 (1995).

[54] H. Fujisaki and K. Nakagawa, Europhys. Lett. 35, 493 (1996).

[55] M.B.C. Abdalla, A.L. Gadelha, and I.V. Vancea, Phys. Lett. A 273, 235 (2000).

[56] I.V. Vancea, Phys. Lett. B 487, 175 (2000).

[57] M.C.B. Abdalla, A.L. Gadelha, I.V. Vancea (unpublished). 